\title{
ON EXPONENTIAL GROWTH RATES FOR FREE GROUPS
}

\author{
MaLiK Koubi
}

\begin{abstract}
Let $F_{p}$ be a free group of rank $p \geq 2$. It is well-known that, with respect to a $p$-element generating set, that is, a basis, the exponential growth rate of $F_{p}$ is $2 p-1$. We show that the exponential growth rate $\tau$ of a group $G$ with respect to a $p$-element generating set $X$ is $2 p-1$ if and only if $G$ is free on $X$; otherwise $\tau<2 p-1$. We also prove that, for any finite generating set $X$ of $F_{p}$ which is disjoint from $X^{-1}$, the exponential growth rate $\tau$ of $F_{p}$ with respect to $X$ is $2 p-1$ if and only if $X$ is a basis of $F_{p}$; otherwise $\tau>2 p-1$.
\end{abstract}

\section{Introduction}

We begin by recalling the basic concepts about exponential growth rate. For details and more information, see the survey article and problem list by R. Grigorchuk and P. de la Harpe [1], and the articles they cite.

Definitions 1.1. Let $G$ be a finitely generated group, and let $X$ be a finite generating set of $G$. For $g \in G$, the $X$-word length of $g,|g|_{X}$, is the least non-negative integer $m$ such that $g$ is the product of a sequence of $m$ elements of $X \cup X^{-1}$. For each non-negative integer $n, \beta_{(G, X)}(n)$ (resp. $\left.\sigma_{(G, X)}(n)\right)$ denotes the number of those $g \in G$ such that $|g|_{X} \leq n$ (resp. $|g|_{X}=n$ ); the letter $\beta$ (resp. $\sigma$ ) refers to the ball (resp. sphere) of radius $n$ centred at 1 . The exponential growth rate of the pair $(G, X)$ is defined as

$$
\tau(G, X)=\lim _{n \rightarrow \infty} \sqrt[n]{\beta_{(G, X)}(n)}
$$

Keywords. Free group, exponential growth rate.

1991 Mathematics subject classifications: Primary: 20E05; Secondary: 20F06. 
We say that $G$ has exponential growth if $\tau(G, X)>1$, a condition which is independent of the choice of finite generating set $X$.

Notice that it is natural to assume that $X \cap X^{-1}$ consists of elements of order two, since we are free to remove elements from $X$ to achieve this condition, without affecting any of the numerical values involved.

Throughout, let $p$ denote an integer such that $p \geq 2$, and $F_{p}$ a free group of rank $p$. It is straightforward to show that if $X$ is a basis of $F_{p}$ then $\beta_{\left(F_{p}, X\right)}(n)=\frac{p(2 p-1)^{n}-1}{p-1}$ and $\tau\left(F_{p}, X\right)=2 p-1$; in particular, $F_{p}$ has exponential growth.

In Section 3, we bound the exponential growth rate from above.

Proposition 1.2. For $p \geq 2$, if $X$ is a p-element generating set of a group $G$, then $\tau(G, X) \leq 2 p-1$, and equality holds if and only if $G$ is free on $X$.

This is reminiscent of the result of Kesten [2], [3], see [1, Section G], that the spectral radius $\mu(G, X)$ of the simple random walk on the Cayley graph of $(G, X)$ is at least $\frac{\sqrt{2 p-1}}{p}$, and equality holds if and only if $G$ is free on $X$. Although it is known that $\mu(G, X) \geq \frac{1}{\tau(G, X)}$, see $[\mathbf{1}$, Section G], it seems to be easier to prove Proposition 1.3 directly, rather than try to derive it from Kesten's result.

Let $X$ be a finite generating set of $F_{p}$ which is disjoint from $X^{-1}$. We shall be interested in $\tau\left(F_{p}, X\right)$. An elementary argument, which we recall below, shows that $\tau\left(F_{p}, X\right) \geq 2 p-1$. Our main result is that equality is achieved if and only if $X$ is a basis of $F_{p}$.

Theorem 1.3. For $p \geq 2$, if $X$ is a finite generating set of $F_{p}$ which is disjoint from $X^{-1}$, then $\tau\left(F_{p}, X\right) \geq 2 p-1$, and equality holds if and only if $X$ is a basis of $F_{p}$.

This will be proved in Section 4. In fact, it suffices to consider the case where $X$ has $p+1$ elements, and we distinguish two cases according to whether $X$ contains a basis of $F_{p}$ or not, and these are dealt with in Lemmas 4.1 and 4.3 , respectively.

\section{Preliminaries}

We now recall the standard results about finitely generated free groups that we shall use. 
Theorem 2.1. Let $n$ be a non-negative integer, and $F_{n}$ a free group of rank $n$.

(i) (J. Nielsen, 1921) Every finite subset of a free group generates a free subgroup.

(ii) (O. Schreier, 1927) If $H$ is a subgroup of finite index $m$ in $F_{n}$ then the rank of $H$ is $m n-m+1$.

(iii) (J. Nielsen, 1921) Every n-element generating set of $F_{n}$ is a basis, so every generating set of $F_{n}$ has at least $n$ elements.

(iv) (folklore) Every generating set of $F_{n}$ contains $n$ independent elements whose images in the abelianization of $F_{n}$ are $\mathbb{Z}$-independent.

(v) (M. Hall, 1949) Every subgroup of $F_{n}$ is a free factor of some subgroup of finite index of $F_{n}$.

Proof: For (i), (ii), (iii), and (v), see Propositions I.2.6, I.3.9, I.2.7, and I.3.10 of [4], respectively.

To see (iv), suppose that $X$ is a generating set of a free group $G$ of rank $n$. Then the image $\bar{X}$ of $X$ in the abelianization $\bar{G}$ is a generating set. But $\bar{G}$ is free abelian of rank $n$, so, by linear algebra, $X$ contains an $n$-element subset $Y$ such that $\bar{Y}$ generates a free abelian subgroup $\bar{H}$ of rank $n$ in $\bar{G}$. Now $Y$ generates a free subgroup $H$ of $G$, by (i). The abelianization of $H$ is free abelian of rank at least $n$, since it maps onto $\bar{H}$, so the rank of $H$ is at least $n$. But $Y$ has $n$ elements, so, by (iii), $Y$ is independent.

In (iv), it would be reasonable to pass from free abelian groups to vector spaces over the field of two elements, but there is no vocabulary to compete with abelianization.

We will find it useful to have a broader scope for the notation given in Definitions 1.1.

Definitions 2.2. Let $G$ be a group and $X$ a finite subset of $G$.

For $g \in G$, we define $|g|_{X}$ to be the least non-negative integer $m$ such that $g$ is the product of a sequence of $m$ elements of $X \cup X^{-1}$, and $\infty$ if no such $m$ exists. For each non-negative integer $n, B_{(G, X)}(n)$ (resp. $\left.S_{(G, X)}(n)\right)$ denotes the set of those $g \in G$ such that $|g|_{X} \leq n$ (resp. $|g|_{X}=n$ ). We write Card to denote the cardinality of a set. As in Definitions 1.1, we set $\beta_{(G, X)}(n)=\operatorname{Card}\left(B_{(G, X)}(n)\right), \sigma_{(G, X)}(n)=$ $\operatorname{Card}\left(S_{(G, X)}(n)\right)$, and

$$
\tau(G, X)=\lim _{n \rightarrow \infty} \sqrt[n]{\beta_{(G, X)}(n)}
$$


Clearly $\tau(G, X)=\tau(\langle X\rangle, X)$, where $\langle X\rangle$ denotes the subgroup of $G$ generated by $X$.

The following is immediate from the definition.

Lemma 2.3. Let $X$ be a finite subset of a group $G$, and let $Y$ be a subset of $X$. Then $\tau(G, Y) \leq \tau(G, X)$.

Proposition 2.4. If $n$ is a non-negative integer, and $X$ is a finite generating set of $F_{n}$, then $\tau\left(F_{n}, X\right) \geq 2 n-1$.

Proof: We may assume that $n \geq 1$. By Theorem 2.1(iv), $X$ contains an $n$-element independent subset $Y$, so $\tau\left(F_{n}, Y\right)=2 n-1$, and, by Lemma 2.3, $\tau\left(F_{n}, X\right) \geq \tau\left(F_{n}, Y\right)=2 n-1$.

\section{Bounding exponential growth rate from above}

We now prove our first result stated in the introduction.

Proposition 1.2. For $p \geq 2$, if $X$ is a p-element generating set of a group $G$, then $\tau(G, X) \leq 2 p-1$, and equality holds if and only if $G$ is free on $X$.

Proof: We may assume that $G$ is not free on $X$, and it remains to show that $\tau(G, X)<2 p-1$.

Thus there is a non-trivial reduced $X$-word $r$ in the free group on $X$, such that $r$ is equal to 1 in $G$. Let $l$ be the $X$-word length of $r$ in the free group on $X$, so $l \geq 1$.

Consider any positive integer $n$.

We claim that

$$
\sigma_{(G, X)}(n+l) \leq\left[(2 p-1)^{l}-1\right] \sigma_{(G, X)}(n) .
$$

To see this, notice that each $h \in S_{(G, X)}(n+l)$ has at least one expression in the form $h=g k$ where $g \in S_{(G, X)}(n)$ and $k \in S_{(G, X)}(l)$, and here there can be no $X$-cancellation. Moreover $k$ cannot be a cyclic permutation of $r$ or $r^{-1}$. Hence, for each $g \in S_{(G, X)}(n)$, there are at most $(2 p-1)^{l}-1$ distinct $k \in S_{(G, X)}(l)$ such that $g k \in S_{(G, X)}(n+l)$. This proves that (1) holds.

For any positive integer $m$, we have

$$
\beta_{(G, X)}(l m)-1=\sum_{i=1}^{l m} \sigma_{(G, X)}(i) \leq \sum_{q=0}^{m-1} \sum_{k=1}^{l}\left[(2 p-1)^{l}-1\right]^{q} \sigma_{(G, X)}(k) .
$$


Now letting $M=\max _{1 \leq k \leq l} \sigma_{(G, X)}(k)$, we see that

$$
\beta_{(G, X)}(l m)-1 \leq l M \sum_{q=0}^{m-1}\left[(2 p-1)^{l}-1\right]^{q}=\frac{M l\left(\left[(2 p-1)^{l}-1\right]^{m}-1\right)}{(2 p-1)^{l}-2} .
$$

Taking $m l$ th roots of boths sides, and letting $m$ tend to $\infty$, we find that $\tau(G, X) \leq \sqrt[l]{(2 p-1)^{l}-1}<\sqrt[l]{(2 p-1)^{l}}=2 p-1$, as desired.

Remark 3.1. In the above proof we obtained the upper bound $\sqrt[l]{(2 p-1)^{l}-1} \geq \tau(G, X)$ in terms of $p$ and the length $l$ of a non-trivial reduced relator in $G$. It is not difficult to show that one can improve this bound replacing $l$ with the greatest integer in $\frac{l}{2}+1$. Notice that $\lim _{l \rightarrow \infty} \sqrt[l]{(2 p-1)^{l}-1}=2 p-1$, which means that we do not obtain any upper bound independent of $l$ better than $2 p-1$.

\section{Bounding exponential growth rate from below}

In this section we prove Theorem 1.3. The case where $X$ contains a basis of $F_{p}$ will be given by the following result.

Lemma 4.1. Let $p \geq 2$. Suppose that $X=\left\{x_{1}, \ldots, x_{p+1}\right\}$ is a generating set of $F_{p}$ such that $Y=\left\{x_{1}, \ldots, x_{p}\right\}$ is a basis of $F_{p}$ and $x_{p+1} \notin Y \cup Y^{-1} \cup\{1\}$. Then $\tau\left(F_{p}, X\right)>2 p-1$.

Proof: Let $a=x_{p+1}$, let $l=|a|_{Y}$, so $l \geq 2$, and let

$$
\gamma=\frac{1}{4 p(2 p-1)^{l-1}}
$$

so $1>\gamma>0$.

Consider any non-negative integer $n$ which is a multiple of the integer $\gamma^{-1}$.

Let $E_{n}=S_{\left(F_{p}, Y\right)}(n l)$, so $a \in E_{1}$ and

$$
\operatorname{Card}\left(E_{n}\right)=2 p(2 p-1)^{n l-1} .
$$

Consider any $w \in E_{n}$. Thus $w$ is a reduced $Y$-word of length $n l$, so is a product of $n$ reduced $Y$-words of length $l$, that is, $w=w(1) w(2) \cdots w(n)$, with each $w(i) \in E_{1}$. 
Let $E_{n}^{\prime}$ be the set of those elements $w$ of $E_{n}$ such that at least $\gamma n$ of the $i$ 's in $\{1, \ldots, n\}$ are such that $w(i)=a$, that is, $a$ "occurs" at least $\gamma n$ times in $w$. Let $E_{n}^{\prime \prime}$ be the complement of $E_{n}^{\prime}$ in $E_{n}$, so $a$ occurs at most $\gamma n$ times in each element of $E_{n}^{\prime \prime}$. Since $a$ occurs at most $n$ times in each element of $E_{n}^{\prime}$, we see that

$$
\sum_{w \in E_{n}} \sum_{i=1}^{n} \delta_{a, w(i)} \leq n \operatorname{Card}\left(E_{n}^{\prime}\right)+\gamma n \operatorname{Card}\left(E_{n}^{\prime \prime}\right),
$$

where $\delta_{x, y}$ is the Kronecker delta function, which is 1 if $x=y$, and 0 otherwise.

For a fixed $i, \sum_{w \in E_{n}} \delta_{a, w(i)}$ is the number of reduced $Y$-words of length $n l$ which have $l$ of the letters fixed, which is easily seen to be $(2 p-1)^{n l-l}$. Hence, the left-hand side of $(3)$ is $n(2 p-1)^{n l-l}$.

Notice that the right-hand side of (3) can be expressed as

$$
(n-\gamma n) \operatorname{Card}\left(E_{n}^{\prime}\right)+\gamma n \operatorname{Card}\left(E_{n}\right)
$$

and this is $(n-\gamma n) \operatorname{Card}\left(E_{n}^{\prime}\right)+\frac{n}{2}(2 p-1)^{n l-l}$ by (2) and the definition of $\gamma$.

Hence (3) becomes $\frac{n}{2}(2 p-1)^{n l-l} \leq(n-\gamma n) \operatorname{Card}\left(E_{n}^{\prime}\right)$, and we have $\frac{(2 p-1)^{n l-l}}{2-2 \gamma} \leq \operatorname{Card}\left(E_{n}^{\prime}\right)$.

Observe that each element of $E_{n}^{\prime}$ can be expressed as a product which has $a$ occurring $\gamma n$ times, together with $(1-\gamma) n$ reduced $Y$-words of length $l$; such an expression has $X$-word length $\gamma n+n l-\gamma n l$, so $E_{n}^{\prime}$ lies in $B_{\left(F_{p}, X\right)}(\gamma n+n l-\gamma n l)$. Hence

$$
\beta_{\left(F_{p}, X\right)}(\gamma n+n l-\gamma n l) \geq \operatorname{Card}\left(E_{n}^{\prime}\right) \geq \frac{(2 p-1)^{n l-l}}{2-2 \gamma} .
$$

Since $2-2 \gamma>0$, taking $\gamma n+n l-\gamma n l$ roots of boths sides, and letting $n$ tend to $\infty$, we see that

$$
\tau\left(F_{p}, X\right) \geq(2 p-1)^{\frac{l}{\gamma+l-\gamma l}}=(2 p-1)^{\left(1-\gamma\left(1-\frac{1}{l}\right)\right)^{-1}}>(2 p-1) .
$$

Remark 4.2. In the above proof we obtained the lower bound $\tau\left(F_{p}, X\right) \geq(2 p-1)^{\alpha(l)}$, where

$$
\alpha(l)=\left(1-\gamma\left(1-\frac{1}{l}\right)\right)^{-1}=\left(1-\frac{1-\frac{1}{l}}{4 p(2 p-1)^{(l-1)}}\right)^{-1},
$$

and $l$ is the $Y$-length of $x_{p+1}$. Since $\lim _{l \rightarrow \infty} \alpha(l)=1$, we do not obtain any lower bound independent of $l$ better than $2 p-1$. 
Lemma 4.3. Let $p \geq 2$, and suppose $X=\left\{x_{1}, \ldots, x_{p+1}\right\}$ is a generating set of $F_{p}$, and that $Y=\left\{x_{1}, \ldots, x_{p}\right\}$ is independent but is not a basis of $F_{p}$. Then $\tau\left(F_{p}, X\right)>2 p-1$.

Proof: Here $\langle Y\rangle$ is free of rank $p$, and is a proper subgroup of $F_{p}$. By Theorem 2.1(ii), $\langle Y\rangle$ has infinite index in $F_{p}$. By Theorem 2.1(v), $\langle Y\rangle$ is a proper free factor of a subgroup of $F_{p}$. In particular, we can extend $Y$ to an independent set $Z=\left\{x_{1}, \ldots, x_{p}, a\right\}$ in $F_{p}$. Let $l=|a|_{X}$. Since $Z$ is independent, $a \notin Y^{ \pm 1}$, and since $X$ is not independent, $a \neq x_{p+1}^{ \pm 1}$. Hence $l \geq 2$.

Consider any non-negative integer $n$.

Let $E_{n}$ denote the set of reduced $Z$-words such that the number of occurrences of each element of $Y^{ \pm 1}$ plus $l$ times the number of occurences of $a^{ \pm 1}$ is $n l$. Notice $E_{n} \subseteq B_{\left(F_{p}, X\right)}(n l)$.

For example, $E_{1}=S_{\left(F_{p}, Y\right)}(l) \cup\left\{a^{ \pm 1}\right\}$, so $\operatorname{Card}\left(E_{1}\right)=2 p(2 p-1)^{l-1}+2$. We will now show that

$$
\operatorname{Card}\left(E_{n}\right) \geq\left[(2 p-1)^{l}+2\right]^{n}
$$

Notice that (3) holds for $n=0$ and $n=1$, so we may assume that $n \geq 2$, and that (3) holds for $n-1$. It suffices to show that each reduced $Z$-word $w \in E_{n-1}$ can be extended in $(2 p-1)^{l}+2$ ways to the right to get an element of $E_{n}$ without any $Z$-cancellation. If $w$ ends in an element of $Y^{ \pm 1}$, we can extend $w$ in $(2 p-1)^{l}$ ways with elements of $S_{\left(F_{p}, Y\right)}(l)$, and in 2 ways with $a^{ \pm 1}$; this is $(2 p-1)^{l}+2$ ways. If $w$ ends in $a^{\epsilon}$, with $\epsilon \in\{ \pm 1\}$, we can extend $w$ in $2 p(2 p-1)^{l-1}$ ways with elements of $S_{\left(F_{p}, Y\right)}(l)$, and in 1 way with $a^{\epsilon}$; here the number of ways is

$$
2 p(2 p-1)^{l-1}+1=(2 p-1)^{l}+(2 p-1)^{l-1}+1 \geq(2 p-1)^{l}+2 .
$$

Hence (3) holds.

Since $E_{n} \subseteq B_{\left(F_{p}, X\right)}(n l),(3)$ implies that

$$
\beta_{\left(F_{p}, X\right)}(n l) \geq\left[(2 p-1)^{l}+2\right]^{n} .
$$

Hence $\tau\left(F_{p}, X\right) \geq \sqrt[l]{(2 p-1)^{l}+2}>\sqrt[l]{(2 p-1)^{l}}=2 p-1$.

Remark 4.4. In the above proof we obtained the lower bound $\tau\left(F_{p}, X\right) \geq \sqrt[l]{(2 p-1)^{l}+2}$, where $l$ is the $X$-length of an element $a$ independent of $Y$. We do not know if there exists a lower bound entirely in terms of $p$ which is greater than $2 p-1$.

We now prove our main result. 
Theorem 1.3. For $p \geq 2$, if $X$ is a finite generating set of $F_{p}$ which is disjoint from $X^{-1}$, then $\tau\left(F_{p}, X\right) \geq 2 p-1$, and equality holds if and only if $X$ is a basis of $F_{p}$.

Proof: We may assume that $X$ is not a basis of $F_{p}$, and it remains to prove that $\tau\left(F_{p}, X\right)>2 p-1$.

By Theorem 2.1(iv), $X$ contains a $p$-element independent subset $Y$ whose image in the abelianization of $F_{p}$ is $\mathbb{Z}$-independent. By hypothesis $X \neq Y$, so there exists a $(p+1)$-element subset $Z$ of $X$ which contains $Y$.

It suffices to show that $\tau(\langle Z\rangle, Z)>2 p-1$, for then, by Proposition 2.4,

$$
\tau\left(F_{p}, X\right) \geq \tau\left(F_{p}, Z\right)=\tau(\langle Z\rangle, Z)>2 p-1
$$

We know that $\langle Z\rangle$ has rank at most $p+1$, and it maps onto a free abelian group of rank $p$, so has rank at least $p$.

If $\langle Z\rangle$ has rank $p+1$ then $\tau(\langle Z\rangle, Z)=2 p+1>2 p-1$. Thus we may assume that $\langle Z\rangle$ has rank $p$.

If $\langle Y\rangle=\langle Z\rangle$ then, since $X$ is disjoint from $X^{-1}$, Lemma 4.1 implies that $\tau(\langle Z\rangle, Z)>2 p-1$. Thus we may assume that $Y$ is not a basis of $\langle Z\rangle$. Here, by Lemma $4.3, \tau(\langle Z\rangle, Z)>2 p-1$. This completes the proof.

Acknowledgements. I warmly thank R. Grigorchuk for the conversations we had in Toulouse and for his interest in the question, and G. Levitt who suggested the problem. I am grateful to the referee for various useful suggestions.

\section{References}

1. R. Grigorchuk and P. De la Harpe, On problems related to growth, entropy and spectrum in group theory, J. of Dynamical and Control Systems 3 (1997), 51-89.

2. H. Kesten, Symmetric random walks on groups, Trans. Amer. Math. Soc. 92 (1959), 336-354.

3. H. Kesten, Full Banach mean values on countable groups, Math. Scand. 7 (1959), 146-156. 
4. R. C. Lyndon And P. E. Schupp, "Combinatorial group theory," Springer, Berlin, 1977.

Laboratoire Emile Picard

CNRS UMR 5580

Université Paul Sabatier

31062 Toulouse Cedex 4

FRANCE

e-mail: koubi@picard.ups-tlse.fr

Primera versió rebuda el 20 de febrer de 1998, darrera versió rebuda el 5 de març de 1998 